Universidad de Medellín

\title{
POLÍTICAS PARA RELACIONES CONTRACTUALES EN LAS CADENAS DE BIOCOMBUSTIBLES: CONSTRUYENDO PUENTES ENTRE INSTITUCIONES*
}

\author{
Recibido: febrero 28 de 2012 - Aceptado: diciembre 11 de 2012 \\ Carlos Eduardo De Freitas Vian** \\ Gustavo Inácio de Moraes ${ }^{* * *}$ \\ Mirian Beatriz Schneider Braun ${ }^{* * *}$
}

\section{RESUMEN}

El objetivo principal de este ensayo reflexivo es analizar los problemas contractuales existentes en las cadenas de biocombustibles en América Latina y el mundo. Se utiliza la estructura organizacional como una unidad de análisis del tema institucional y se asume como algo endógeno, es decir, como algo sobre el cual los agentes influyen para validar sus intereses y estrategias. Las evidencias encontradas permiten plantear algunas directrices de política que pueden contribuir a mejorar las relaciones contractuales en las cadenas de biocombustibles.

\section{PALABRAS CLAVE}

Biocombustibles, cadenas industriales, América Latina, políticas públicas, sustentabilidad

\section{CLASIFICACIÓN JEL}

L7, 054, O14, Q16, Q28

\section{CONTENIDO}

Introducción; 1. Panorama de las políticas de biocombustibles en el mundo: factores determinantes y niveles de implementación; 2. Acciones y propuestas motivadoras para biocombustibles; 3. Problemas de coordinación en las cadenas productivas de biocombustibles; 4. Experiencias de coordinación de las cadenas y sus posibles lecciones; 5 . Consideraciones finales sobre las directrices de políticas para mejoras en las relaciones contractuales; Bibliografía

\footnotetext{
Reflexión sobre las cadenas industriales en América Latina evaluada en Grupo de Extensión y Pesquisa en Historia de la Agricultura y de los Complejos Agroindustriales (GEPHAC), de la Universidad de San Paulo (USP), Brasil. Los dos primeros autores presentaron una versión preliminar del documento en Congreso Brasileiro de Economía Rural, (SOBER), 2008, en Rio Branco - Acre.

." Economista, Universidad Estatal de Campinas (UNICAMP), Brasil. Doctor en Economía, Universidad Estatal de Campinas, Brasil. Profesor del Departamento de Economía, Administración y Sociología, Escuela Superior de Agricultura Luiz de Queiroz (ESALQ), Universidad de Sao Paulo (USP), Brasil. Coordinador del Grupo de Extensión e Investigación en Historia de la Agricultura y de los Complejos Agroindustriales (GEPHAC) y del Grupo de Estudios y Extensión en Desarrollo Económico y Social (GEEDES), ESALQ, USP, Brasil. Correo electrónico: cefvian@esalq.usp.br.

*.* Economista, Universidad de Sao Paulo, Brasil. Maestría en Desarrollo Económico Universidad Federal de Paraná, Brasil. Doctor en Escuela Superior de Agricultura Luiz de Queiroz, Universidad de Sao Paulo, Brasil. Profesor de Posgrado en Economía, Pontificia Universidad Católica de Rio Grande del Sul (PUCRS), Brasil. Correo electrónico: gustavo.moraes@pucrs.br.

… Economista, Universidad Estatal del Oeste de Paraná (UNIOESTE), Brasil. Maestría en Desarrollo Económico, Universidad Federal de Paraná, Brasil. Doctora en Historia Económica, Universidad de León, España. Profesora, Departamento de Economía, UNIOESTE, Campus de Toledo, Paraná, Brasil. Investigadora del Grupo GEPEC. Correo electrónico: mirianbraun@unioeste.br.
} 


\section{POLICIES TO CONTRACT RELATIONS IN BIOFUELS INDUSTRY: BUILDING BRIDGES BETWEEN INSTITUTIONS \\ ABSTRACT}

The aim of this paper is to analyze organizational problems in biofuels industry in Latin America context and propose public policies, specially seeking for improve relationships among different actors, role of contracts and better instruments, and in consequence provide sustainability in the production and consumption of renewable fuels. Organizational field is the unit of analysis of institutional matter like endogenous or a place where strategies and interests are opposed. In this paper, a description of the major biofuels programs in Brazil, and your contrast against worldwide programs, offers insights in supply hub. Finally, some guidelines in public policies that can better contractual relations in biofuels industry are presented.

\section{KEY WORDS}

Biofuels, Industry relations, Latin America, Public Policies, Sustainability.

\section{JEL CLASSIFICATION}

L7, O54, O14, Q16, Q28

\section{CONTENT}

Introduction; 1. Overview in world policies for biofuels: determinant factors and acting levels. 2. Actions and motivating proposals for biofuels; 3. Coordination problems in biofuels industry; 4. Coordination experience in industry and possible lessons; 5 . Final comments about policies for better contract relations; Bibliography.

\section{POLITICAS PARA RELAÇÕES CONTRATUAIS NAS CADEIAS DE BIO-COMBUS- TÍVEIS: CONSTRUINDO PONTES ENTRE INSTITUIÇÕES \\ RESUMO}

O objetivo principal deste texto é analisar os problemas contratuais existentes nas cadeias de biocombustíveis na América Latina e propor diretrizes de políticas, instrumentos e o papel dos diferentes agentes, em busca de melhorar estas relações e dar confiabilidade e sustentabilidade a produção e ao consumo de combustíveis renováveis. Indica-se o Campo Organizacional como uma unidade de análise da questão institucional como algo endógeno, ou seja, como algo sobre a qual os agentes influenciam seus interesses e estratégias. Apresenta-se, em um ensaio reflexivo, uma descrição dos principais programas de biocombustíveis na América Latina e no mundo e, também, os problemas contratuais nas cadeias. Por fim, serão apontados algumas diretrizes de políticas que podem melhorar as relações contratuais nas cadeias de biocombustívies.

\section{PALAVRAS CHAVE}

Biocombustíveis, cadeias industriais, América Latina, políticas públicas, sustentabilidade.

\section{CLASSIFICAÇÃO JEL}

L7, 054, O14, Q16, O28

\section{CONTEÚDO}

Introdução; 1. Panorama das Políticas de biocombustíveis no mundo: factores determinantes e níveis de implementação; 2. Ações e propostas motivadoras para biocombustiveis; 3. Problemas de coordenação nas cadeias produtivas de biocombustíveis; 4. Experiências de coordenação das cadeias e suas possíveis lições; 5. Considerações finais sobre as diretrizes de políticas para melhorias nas relações contratuais; Bibliografia 
Políticas para relaciones contractuales en las cadenas de biocombustibles: construyendo puentes entre instituciones

\section{INTRODUCCIÓN}

En los últimos años, el interés por la producción y uso de biocombustibles creció en todo el mundo. Uno de los principales motivos es la polución generada por el uso de combustibles fósiles, principalmente en las grandes ciudades. Otro factor relevante es la tendencia de crecimiento del precio del petróleo motivado por el alto consumo y por los problemas sociales y políticos en algunas de las principales regiones productoras. Muchos países lanzaron programas de sustitución de gasolina y diésel por biocombustibles y han creado incentivos para aumentar la producción interna de los mismos. En muchos casos esta producción no existía y la oferta de materia prima era pequeña o nula; tampoco existía presencia de cadenas de producción y distribución de biocombustibles.

La preocupación por las externalidades negativas asociadas a los combustibles fósiles asumió proporciones importantes a mediados de los años ochenta. El crecimiento del movimiento ecológico y la presencia de los temas en las agendas políticas hicieron que el tema tomara mayor relevancia. La consolidación del concepto de desarrollo sustentable fue apenas parte de un proceso que se fortaleció con la adopción formal de políticas ambientales de todos los gobiernos mundiales en los años noventa. Estos aspectos institucionales pasaron a moldear las estrategias de las empresas y las relaciones con proveedores, empleados, clientes y sociedad en general.

De forma paralela, se constató que la humanidad ya podría haber alterado el ambiente del planeta de forma definitiva. El crecimiento económico anterior se dio sobre una base energética limitada y frágil, y la humanidad constata un cambio climático que tiene como fuente las actividades humanas.

Dentro de este contexto, una transición dentro de la matriz energética ${ }^{1}$ es necesaria y los denominados biocombustibles asumen un papel importante para suplir la demanda de un crecimiento económico continuo y paralelamente ofrecer soluciones para una serie de problemas que el crecimiento económico produce.

De manera particular, los biocombustibles ofrecen oportunidades para regiones aún no desarrolladas y para ocupar parte de la mano de obra no calificada y, además, ofrecen una fuente energética que permite reducir las externalidades negativas producidas por las fuentes tradicionales de energía, principalmente, el nivel de emisión de gases. Por otro lado, la producción de biocombustibles ha sido identificada como una amenaza a la producción de alimentos, en la medida en que competiría por el uso de las tierras. Además, se hicieron evidentes limitaciones en la

Se entiende por Matriz Energética el conjunto de todas las diferentes fuentes de generación de energía.

Semestre Económico, volumen 16, №. 33, pp. 15-44 • ISSN 0120-6346, enero-junio de 2013, Medellín, Colombia 
infraestructura de distribución y en la garantía de una producción libre de estacionalidades, fundamental para la adopción por parte de los consumidores. Además, se puede citar que las cadenas productivas de biocombustibles están formándose a partir de otras, consolidadas en función de la producción de alimentos para la producción de energía.

El presente texto tiene como objetivo principal discutir las políticas adoptadas para biocombustibles en diferentes países del mundo, pero principalmente en América Latina. Como objetivos secundarios el artículo discute la necesidad de mejorar las relaciones industriales en articulación y las políticas públicas relacionadas con las relaciones contractuales en las cadenas de biocombustibles.

Para alcanzar los objetivos del artículo, se optó por elaborar un ensayo reflexivo, dialogando con la literatura acerca del tema y presentando, en especial, la situación brasileña. El artículo está dividido en cinco sesiones.

En la primera, se presenta el panorama mundial de los biocombustibles y especialmente la adopción de estímulos para la industria. En la segunda, parte se presenta la justificación para el uso de biocombustibles y su adopción. En la tercera, se abordan las dificultades de coordinación en la compleja cadena de biocombustibles. En la cuarta, se discuten las posibles lecciones de las experiencias brasileñas. Finalmente, en la quinta sección, se presentan los comentarios y recomendaciones de los autores.

\section{PANORAMA DE LAS POLÍTICAS DE BIOCOMBUSTIBLES EN EL MUNDO: FACTORES DETERMINANTES Y NIVELES DE IMPLEMENTACIÓN}

Según Hobsbawn (1995) y Heilbroner (1996), el crecimiento económico a partir de la Revolución industrial se vio favorecido por el uso de las fuentes energéticas densas, como el carbón y el petróleo. Esas fuentes, de origen fósil, producen externalidades negativas: la contaminación, notada por varios observadores desde los primeros tiempos del uso de estas fuentes.

Con la llegada del siglo XX y con el surgimiento de Rusia y Estados Unidos en el escenario mundial, el petróleo pasó a ocupar un lugar destacado en la matriz energética mundial. Para Martin (1989), esa transición ocurrió, sobre todo, al final de la Segunda Guerra Mundial; bajo el contexto de las inversiones del Plan Marshall y la industrialización del Este europeo, las plantas industriales de los países europeos adquirieron como materia prima principal los derivados del petróleo.

El primer problema para el abastecimiento estable del petróleo ocurre en la década de 1970 con el control de precios, establecido por la OPEP, principalmente 
Políticas para relaciones contractuales en las cadenas de biocombustibles: construyendo puentes entre instituciones

los países árabes. El segundo problema de abastecimiento de petróleo surgió por el aumento del conocimiento sobre los efectos negativos del consumo del petróleo y sus derivados. La conceptualización del desarrollo sustentable a partir del Documento Brutland, CMDE (1991), y la conferencia Río 92, situaron el problema en un nuevo trípode político.

La percepción respecto a los cambios climáticos y su probable causa antropogénica realzó el desafío impuesto a la formulación de políticas para el desarrollo de nuevas fuentes energéticas. En este sentido, la política óptima está obligada a mantener el crecimiento económico, sin dejar de lado la transición energética, rumbo a unas fuentes energéticas que proporcionen menor nivel de emisión de contaminantes; al mismo tiempo, aún mejor, si es posible, en este proceso lograr que se corrija algunas desigualdades originadas en el proceso de crecimiento económico.

Antes del reciente boom de los biocombustibles, iniciado alrededor del año 2002, experiencias relacionadas con la producción y utilización de etanol pueden ser encontradas en el continente americano (Brasil ${ }^{2}$, Argentina, y Costa Rica) y en el continente africano (Kenia, Malawi, Zimbaue y Suazilandia), experiencias que prueban que los incentivos estatales dirigidos a los programas de sustitución de combustibles fósiles fueron exitosos. De acuerdo con Gowen (1989), los biocombustibles requieren una alta inversión inicial y generan altos costos de transporte, obstáculos que ya eran perceptibles a finales de la década de los ochenta. A estos factores se debe agregar la necesidad de consolidar las relaciones entre los eslabones de la cadena productiva, desarrollo y difusión de tecnología, entre otros aspectos.

Para Canadá, México y Estados Unidos, el consumo de biomasa en la totalidad de la matriz energética aún se encontraba en bajos niveles a comienzos de los años noventa y, en su mayoría, era consumida por el sector industrial. Las políticas para el consumo de biomasa como fuente energética fueron iniciadas en 1978 en Canadá, para lo cual fue necesario recurrir a importaciones de biomasa; simultáneamente, aplicaron estímulos a la investigación tecnológica y al desarrollo interno de la producción. Según Klass (1995), en Estados Unidos las primeras leyes que crearon incentivos para generación de energía derivada de biomasa se establecieron en 1978. Estas estrategias fueron abandonadas durante los años ochenta y volvieron a ser retomadas a partir de 1992, a través de la creación de incentivos fiscales para el consumo y la investigación.

En Canadá un programa de adopción de etanol a base de maíz fue instituido en 2003 y reforzado con incentivos fiscales a partir de 2006, programa que bus-

2 De acuerdo con Leite (1997), a partir de la década de 1920. 
caba que el consumo alcanzara el equivalente a 5 \% de los combustibles en 2010. De acuerdo con Forge (2007), paralelamente, fueron programados subsidios para finqueros, investigación y desarrollo, con el fin de garantizar una oferta firme del producto. Para Forge (2007, p. 5), las necesidades de investigación y desarrollo eran urgentes, ya que para lograr cubrir el $10 \%$ del consumo de combustibles para transporte, Canadá necesitaba plantar 36 \% de su área arable, mientras que Brasil apenas necesitaba destinar un $3 \%$ del área arable.

Bernard y Prieur (2007) plantean que en la Unión Europea, además de la importancia política que tiene el asunto de los cambios climáticos, la preocupación central gira en torno al sector de transportes, responsable del $67 \%$ del consumo de petróleo en la región. De esta forma, la política de energertica se preocupa por garantizar el suministro de biocombustibles del sector de transportes lo que le pueden permitir evitar las costosas importaciones de petróleo, los recursos destinados a estas importaciones son vistos como la razón principal para justificar los subsidios agrícolas y logar la seguridad energética.

Como muestra Faaij (2006), la expansión de esa fuente energética desde mediados de los años noventa en la Unión Europea concentra alrededor de 9 \% en la generación de electricidad. Asimismo, se evidencia que el desarrollo de la utilización de biomasa, por la experiencia europea, pasa por seis fases: la primera es la sistematización de la recolección de residuos; la segunda fase es la utilización local de recursos agrícolas y forestales, con el consecuente desarrollo de infraestructura; la tercera fase es el aprovechamiento de economías de escala en el transporte, desarrollo de un mercado regional y mayor capacidad de procesamiento, aliada a una conversión más eficiente; la cuarta fase comprende el desarrollo de mercados nacionales y la integración de la logística; puede ser posible en esta fase observar bajas de precios; en la quinta fase, el mercado crece más allá de las fronteras y surgen los primeros conflictos respecto al comercio internacional, incluyendo cuotas y subsidios; finalmente, en la sexta fase, el mercado se consolida con plantíos dedicados exclusivamente a la bioenergía.

Jull y otros (2007) realizaron una investigación sobre los instrumentos institucionales utilizados en la promoción de biocombustibles alrededor del mundo. La principal preocupación referente al comercio internacional sigue siendo un acuerdo que logre determinar los subsidios y tarifas de protección para la agricultura, y que, como consecuencia, posibilite un comercio mayor de etanol y biocombustibles. Los combustibles renovables aún son considerados productos industrializados, aunque su materia prima sea agrícola. Con respecto a los países de América Latina, el estudio plantea que la mayoría ha optado por ceder la administración de la política de biocombustibles a un órgano creado exclusivamente para ese fin. 
Políticas para relaciones contractuales en las cadenas de biocombustibles: construyendo puentes entre instituciones

Una economía abastecida por biocombustibles, aunque disminuya la intensidad de contaminación, también presentará algunas externalidades. La FAO (2007) alerta sobre el efecto de la producción de biocombustibles en la seguridad alimenticia, en la medida en que la producción de estos puede encarecer el precio de los alimentos por causa de la competencia de uso de tierras, nuevos usos para ciertos productos agrícolas e impactos ambientales originados por las nuevas variedades transgénicas (soya y maíz).

Existe un reconocimiento de esta externalidad sobre el precio de los productos agropecuarios en Kruse y otros (2006). La eliminación de los subsidios internos y el aumento de las alícuotas de importación podrían, además de desestimular la producción de biocombustibles, reducir la renta del sector agropecuario americano. Según el estudio, para el período comprendido entre 2011 y 2016 el sector agropecuario perdería cerca de US\$ 3 billones por año, lo que justifica que se continúe manteniendo las ventajas internas (subsidios) para los productores de maíz.

La necesidad de tierras para la producción de biocombustibles a partir de la caña en la India es evaluada por Sudha y Ravindranath (1999), quienes destacan un escenario donde el país se convierte en importador de alimentos entre los años 1990 y 2030, en función de su crecimiento poblacional; se calcula que el área disponible para la producción de biomasa sería de 43 millones de hectáreas. La mayor parte se localiza dentro de las zonas agroecológicas (26 millones) y la menor parte en áreas que no compiten con la producción de alimentos ( 3 millones en pastizales y 14 millones en áreas no disponibles para cultivo). La producción total de biomasa dependería de la productividad que se espera alcanzar.

En Francia, la adopción de los biocombustibles sigue la orientación de la política establecida por la Unión Europea. Bernard y Prieur (2007) señalan que de acuerdo con las metas trazadas por el Gobierno francés, habría necesidad de una ampliación considerable de áreas ocupadas con cultivos encaminados a la producción de biocombustibles. De esta manera, se considera inevitable la competencia entre áreas dedicadas a la agricultura y áreas dedicadas a la agroenergía. La necesidad de áreas adicionales crecería, sobre todo después del 2008, cuando las metas se vuelven más ambiciosas. Al mismo tiempo, indican que la taza de exención para los biocombustibles podría ser menor, en la medida en que los precios del petróleo se mantengan en niveles elevados.

Berndes y Prieur (2007) sintetizan la necesidad de tierras en el contexto europeo para la producción de los biocombustibles, dividiendo el continente entre los países del Este europeo ${ }^{3}$ y los quince que constituían la Unión Europea hasta el 2003. En

3 CEEC - Central and Eastern European Countries. 
los primeros, estiman un área de 2 millones de hectáreas en 2010, mientras que en los últimos el área alcanzaría 8 millones de hectáreas, o 10 \% del área cultivable y área de cultivo permanente. En el 2020 y 2030, el porcentual de ocupación de estas áreas saltaría a 30 y $40 \%$, respectivamente, en las dos regiones consideradas. El costo de producción todavía sería mayor en la región de la Unión Europea. Se debe destacar que los países del Este poseerían condiciones para tornarse exportadores de biocombustibles al resto de Europa, además de tener una capacidad mayor de absorción de mano de obra en su producción.

En Italia, se calculó que un tercio del área disponible para la agricultura sería necesaria para que se alcancen los objetivos propuestos por la Unión Europea. Los impactos, además de la elevación de los precios de los productos agropecuarios, serían la mayor importación de alimentos, con repercusión sobre la cuenta corriente, y también la reducción de la facturación del país por energía, en caso del establecimiento de subsidios. Por otra parte, Russi (2008) plantea que el desarrollo de las áreas rurales en el contexto de producción de biocombustibles podría ser afectado con otras políticas como, por ejemplo, el desarrollo de productos orgánicos.

Para el territorio de la Republica Checa, Lewandowski y otros (2006) plantean que la producción de biocombustibles en 2030 será capaz de abastecer la demanda interna y exportar una gran cantidad a la Unión Europea. El estudio de la productividad agrícola y la disponibilidad de tierras constituyen las variables decisivas para determinar la capacidad de producción y el éxito de la estrategia. Un aumento en la capacidad de conversión sería importante para requerir un área de producción menor, factor que está ligado al desarrollo de nuevas tecnologías.

Frente a los problemas señalados, para viabilizar el uso de los combustibles se requiere de una mejor planificación. Por eso en la próxima sección se discute cuáles son las acciones que auxiliarían la conquista de esa diversificación en combustibles y cuáles propuestas pueden tornarse viables.

\section{ACCIONES Y PROPUESTAS MOTIVADORAS PARA BIOCOMBUSTIBLES}

Las propuestas para viabilizar los biocombustibles y la superación de los principales problemas asociados a su adopción se pueden dividir en tres categorías de acción: una para avances tecnológicos, con los que se busca principalmente el aumento de la eficiencia; una segunda asociada a medidas institucionales, tales como incentivos y subsidios públicos y/o reformas de los códigos públicos, como el tributario; la tercera, asociada con el análisis de las características productivas de cada país y región, que busca implementar las políticas con enfoque en los puntos fuertes de cada una. 
Políticas para relaciones contractuales en las cadenas de biocombustibles: construyendo puentes entre instituciones

Al analizar la región de Toscaza -Italia-, Bernetti, Fagarazzi y Frantini (2004) destacan el potencial de aprovechamiento de las áreas montañosas de la localidad como base de producción de materias primas para los biocombustibles, sobre todo, derivados de los bosques de la región. Uno de los méritos de este trabajo es evaluar la viabilidad económica de las empresas hipotéticas instaladas para la producción de materia prima. Los efectos macro económicos se reflejarían en el aumento del empleo y de nuevas inversiones.

Otro país preocupado por incrementar la utilización de biocombustibles es Alemania. Así como en otros países, principalmente Estados Unidos, existen cooperativas de producción que conviven con grandes productores individuales. La reciente adopción de metas con plazos para los años de 2010 y 2015 da la seguridad de la presencia de inversiones nuevas, ya que la actual capacidad no sería suficiente para el cumplimiento de las metas establecidas. Según Dautzenberg y Honf (2008), la coordinación entre los diferentes niveles de producción (hacendados y procesadores) sería un prerrequisito necesario, y la principal acción, en este momento de expansión. Para los hacendados, la cooperación pasa por la garantía del precio, al paso que para el procesamiento, la reducción de costos de transporte es el tema principal. La garantía de precio podría ser facilitada con la creación de mercados de futuros de las materias primas de biocombustibles, algo perfectamente operacional en estos días.

Se debe resaltar que en el caso americano, además de las negociaciones de precio, las fábricas de alcohol de maíz son compañías abiertas, y que los agricultores, transportadores y otros agentes de la cadena son dueños de acciones. De este modo, el diálogo existe y se forma un campo organizacional relativamente estable.

En el contexto sueco, Brannlund y Kristrom (2001) examinan, a partir de la función de producción estimada para fábricas que tienen por objetivo el calentamiento, los efectos de impuestos adicionales. El programa sueco de incentivos a los biocombustibles, iniciado en 1997, usa los impuestos e incentivos financieros, con el propósito de lograr menores niveles de emisiones. Como constatan los autores, el programa todavía debería focalizar nuevos esquemas tributarios. Consideran ineficiente utilizar límites de emisiones asociados a esquemas tributarios.

Con relación a la aplicación de subsidios y tributos para el estímulo de los biocombustibles, Rozakis, Sourie y Vanderpooten (2001) estudian los impactos en Francia, teniendo en cuenta tanto el lado de la oferta como el de la demanda. A través de modelos de programación lineal, los autores observan que frente a tres objetivos (garantizar un precio adecuado a los productores primarios, mejores niveles de exención y configuración de técnicas adecuadas) los niveles subsidios y tributos 
fijados por el Gobierno francés serían mayores que los considerados óptimos. Los niveles de exención serían todavía menores, si se considera como objetivo principal el gasto público, sumándose a los demás objetivos.

Costa (2004) recuerda que para que los proyectos de cultivos encaminados a la producción de biocombustibles sean viables serán necesarios algunos esfuerzos para el desarrollo de investigaciones adicionales, sobre todo las ligadas al alcance de mayor productividad y a la resolución de las disparidades regionales. Macedo (2007), por ejemplo, relata que la productividad de las labores de caña de azúcar, utilizadas para la producción de etanol en el centro-sur de Brasil era de 84,3 toneladas por hectárea para 2003-2004. Con todo, la productividad de caña de azúcar en otras áreas de Brasil y en la propia región centro-sur puede ser mayor y frecuentemente ha sido estimada como mayor ${ }^{4}$.

En Turquía, los cultivos que presentaron mayor poder calorífico, en orden, fueron: maíz, trigo y algodón. Además se calculó que el potencial de generación a través de los residuos agropecuarios y animales representaría hasta el 27 \% del consumo energético del país. Oztertuk y Bascetincelik (2006) argumentan que incentivos o reglas que estimulen el uso de ese potencial aún son mínimos y podrían ser orientados al uso en pequeñas escalas. Para Tesalia, región agrícola de Grecia, el potencial de producción de agroenergía es modelado según Rozakis y Sourie (1998).

El aprovechamiento de áreas degradadas en bosques tropicales también requiere del apoyo gubernamental, ya que están distantes de los centros consumidores, necesitarán de crédito inicial para la implementación y tendrán que garantizar una producción continua, lo que hace necesario y prioritario el diseño de estrategias para administrar la estacionalidad. En estos casos, la producción para uso local sería la mejor alternativa, dado que induce el desarrollo regional.

Al respecto, FAO (2007) indica la posibilidad del uso de áreas inclinadas y de mosaicos, normalmente no aprovechados para la producción de alimentos. Además, la producción de biocombustibles en esas áreas contribuiría a mejorar la infraestructura local; de forma paralela, el establecimiento de zonas agroecológicas, junto a una rotación de producción, a veces enfocada hacia la bioenergía, a veces hacia los alimentos, en tierras que demuestren ser viables para las dos producciones. En este rol de recomendaciones es importante que también se acompañe la evolución de la productividad agrícola, cuyas mejoras pueden hacer disminuir la necesidad de expansión adicional.

Ver Marín y otros (2007). 
Políticas para relaciones contractuales en las cadenas de biocombustibles: construyendo puentes entre instituciones

Es necesario considerar que en las negociaciones internacionales de comercio, los países en desarrollo deben tener la capacidad para demostrar que la producción de biocombustibles no avanzará o pone en riesgo las tierras hoy ocupadas por bosques. Adicionalmente, la liberación del comercio internacional de biocombustibles y sus respectivas alícuotas podrán ejercer un papel preponderante en la decisión de nuevas inversiones y, como consecuencia, en la especialización productiva de algunas regiones.

Los combustibles originados a partir de la biomasa han ocupado un lugar destacado en Blangadesh, al ubicarse en un segundo lugar después de fuentes como la energía solar y la hidroelectricidad. Islam, Islam y Rahman (2006), destacan que la instalación de un horno, con rendimiento superior, en escala comercial resulta viable en las áreas rurales.

Bathia (1985) ya destacaba que dentro de la agricultura del subcontinente asiático, una importante cuota de energía requerida era producida en la propia unidad de producción a mediados de los años setenta, donde los residuos y la fuerza animal eran los principales responsables de esa generación de energía. Entretanto, la introducción de técnicas mecanizadas contribuyó a la disminución reciente de esta producción autónoma de energía.

Schell, Riley y Petersen (2008) destacan que en el proceso industrial de procesamiento de biocombustibles pueden surgir subproductos, que crean la posibilidad de agregar un mayor valor. Esfuerzos adicionales en investigación y desarrollo deberán ser realizados, lo que creará viabilidad operacional vía adaptación en las unidades consumidoras, y un transporte adecuado, sea cual fuere el país o la región considerada.

Ometto, Ramos y Lombardi (2007) señalan que muchas de las externalidades negativas en la producción de etanol en Brasil han sido resueltas a través de la integración de unidades productoras. La integración de la cadena productiva y la producción conjunta de biocombustibles, alimento y electricidad presentan un retorno estimado de cinco años.

En Cuba, el aprovechamiento de los residuos de la caña de azúcar aún es relativamente incipiente. Pippo, Garzone y Cornacchia (2007) estiman que la tonelada de bagazo podría alcanzar US\$27.7, mientras que para todos los residuos combinados de caña, la tonelada llegaría a US\$22.86. Concluyen que las inversiones para la cogeneración y el aprovechamiento de este potencial son demasiado altas y el retorno amplio. Uno de los problemas que permanecen es la baja cantidad producida fuera de la época de cosecha, lo que genera poca seguridad en la utilización de este 
tipo de energía en el país. De este modo, cualquier sistema energético capaz de aprovechar el potencial de los residuos dependería de los subsidios para ser viable.

Hagerdal y otros (2006) también apuntan la necesidad de avances técnicos para que la oferta de combustibles fundamentados en biomasa permanezca firme. Entre estos avances está el perfeccionamiento de la tecnología de fermentación, del metabolismo, de la enzima y del proceso general de producción. La biomasa derivada de la lignocelulosa también podrá ser aprovechada a escala industrial.

Lang y otros (2001) señalan como una ventaja la menor volatilidad de las propiedades de los biocombustibles frente al diésel. El estudio plantea que los biocombustibles, en general, a partir de cualquier planta, tienen propiedades compatibles con el diésel. Aún permanece el problema del uso de biocombustibles sobre condiciones de clima extremadamente frío, pero aún es posible superar el problema por medio de la complementación por aditivos.

La asociación de productores en esquemas cooperativos, con nuevas configuraciones frente a nuevos procesos y nuevos productos, puede generar ventajas expresivas. Downing, Volk y Schimidt (2005) evalúan los esquemas cooperativos a través de los precios, producción y conducta y sugieren que en el campo de la bioenergía todavía existe espacio para el desarrollo de actividades cooperativas.

La energía que proviene de la biomasa ya representa una cuota importante de la matriz energética de los países en desarrollo y, especialmente de las áreas rurales. La producción local de biocombustibles aumenta las ventajas competitivas en la utilización de estos en las áreas rurales, desde que la implementación técnica sea la adecuada y cuente con auxilio financiero externo a la región. Demirbas y Demirbas (2007) plantean que esto es válido para el continente asiático y el africano, aunque en las áreas más pobres de América este argumento también es válido. Se debe destacar que muchas de las políticas de biocombustibles no dan importancia a la cuestión local y regional, y sí a la sustitución de combustibles en la matriz de transporte nacional.

Faaji (2006) argumenta que los objetivos a ser perseguidos en el futuro incluyen permanente desarrollo de opciones tecnológicas y la creación de un mercado internacional de biomasa.

Jull y otros (2007) también destacan la necesidad de garantizar disponibilidad adecuada de materias primas, y su análisis se concentra en los instrumentos institucionales. La coordinación entre los diversos eslabones de la cadena debe ser anclada, según el estudio, por medio de amarras institucionales. Otro avance 
Políticas para relaciones contractuales en las cadenas de biocombustibles: construyendo puentes entre instituciones

posible sería la certificación de la producción de biocombustibles, al mostrar el uso de prácticas ambientales, laborales y de gestión adecuadas, que agregan valor en el momento de la comercialización.

El aumento planificado del consumo de biomasa como combustible en los países de la Unión Europea lleva a Mathews (2007) a concluir que la producción de biomasa de Brasil sería insuficiente, y que será necesario incrementar la producción en un equivalente superior a diez veces la producción de Brasil. Más que las fuerzas de mercado, serán necesarias herramientas que posibiliten el acceso a los mercados de los países en desarrollo, con el objetivo de mantener un abastecimiento regular. Por tanto, la creación de un mercado de agenda exige el establecimiento de aquello que el autor llama de "bioimpacto".

Desde el punto de vista del transporte de las áreas exportadoras de energía hacia las áreas demandantes, se hace necesaria una logística adicional para evitar pérdidas y reducir el costo. De esta forma, la posibilidad de uso local sería reducida al crear un mercado. En este sentido, Hamelinck, Suurs y Faaij (2005) plantean que la producción suramericana, llevada a cabo con menor costo, posee ventajas con relación a, por ejemplo, la producción norteamericana y del Este europeo.

El cuadro 1 sintetiza las políticas adoptadas hasta ahora para la producción y uso de biocombustibles en el mundo. Debe señalarse que las mismas tienen un carácter estrictamente económico y no dan importancia a características regionales y locales, como creación de cadenas locales de producción y consumo, sustitución de diésel para generación de energía eléctrica y otras formas de inserción en el mercado nacional, entre otras.

Puede también destacarse que no hay preocupación en cuanto a la creación de incentivos a diferentes formas de coordinación a lo largo de las cadenas productivas, dado que se pueden usar incentivos tradicionales, como los precios tabulados, e incentivos fiscales, que no son suficientes para garantizar el abastecimiento de biocombustibles, pues existe la competencia de usos alternativos, como la producción de alimentos, usos industriales de ciertos aceites, entre otros aspectos.

Cuadro 1. Síntesis de políticas para biocombustibles en países seleccionados

\begin{tabular}{|l|c|c|c|c|c|}
\hline \multicolumn{1}{|c|}{ PAÍs } & $\begin{array}{c}\text { Impuestos / } \\
\text { Subsidios }\end{array}$ & $\begin{array}{c}\text { Programas en } \\
\text { desarrollo }\end{array}$ & $\begin{array}{c}\text { Producción } \\
\text { Agrícola }\end{array}$ & $\begin{array}{c}\text { Logística de } \\
\text { Comercialización }\end{array}$ & $\begin{array}{c}\text { Inversiones en } \\
\text { I+D }\end{array}$ \\
\hline Brasil & Sí & Sí & Sí & Incipiente & Sí \\
\hline Argentina & Incipiente & Sí & Sí & Incipiente & Sí \\
\hline
\end{tabular}

Semestre Económico, volumen 16, №. 33, pp. 15-44 • ISSN 0120-6346, enero-junio de 2013, Medellín, Colombia 
Carlos Eduardo De Freitas Vian • Gustavo Inácio De Moraes • Mirian Beatriz Schneider Braun

\begin{tabular}{|c|c|c|c|c|c|}
\hline PAÍS & $\begin{array}{l}\text { Impuestos / } \\
\text { Subsidios }\end{array}$ & $\begin{array}{c}\text { Programas en } \\
\text { desarrollo }\end{array}$ & $\begin{array}{l}\text { Producción } \\
\text { Agrícola }\end{array}$ & $\begin{array}{c}\text { Logística de } \\
\text { Comercialización }\end{array}$ & $\begin{array}{c}\text { Inversiones en } \\
\mathrm{I}+\mathrm{D}\end{array}$ \\
\hline Costa Rica & Sí & Sí & Sí & Incipiente & No \\
\hline Kenia & No & No & Sí & No & No \\
\hline Malawi & No & No & Sí & No & No \\
\hline Zimbabwe & No & No & Sí & No & No \\
\hline Suazilandia & No & No & Sí & No & No \\
\hline Francia & Sí & Sí & Sí & Sí & Sí \\
\hline Alemania & Sí & Sí & Sí & Sí & Sí \\
\hline Italia & Sí & Sí & Sí & Sí & Sí \\
\hline Suecia & Sí & Sí & No & Sí & Sí \\
\hline Reino Unido & Incipiente & Sí & No & Sí & Sí \\
\hline Holanda & Incipiente & Sí & No & Sí & Sí \\
\hline Rep. Checa & Sí & Sí & Sí & Incipiente & Incipiente \\
\hline Hong Kong & Sí & No & No & Sí & No \\
\hline Canadá & Sí & Sí & Sí & Sí & Sí \\
\hline México & Sí & Sí & Sí & Incipiente & Incipiente \\
\hline EUA & Sí & Sí & Sí & Sí & Sí \\
\hline India & Incipiente & Sí & Sí & Incipiente & Incipiente \\
\hline Cuba & No & No & Sí & No & No \\
\hline
\end{tabular}

Fuente: Datos de la investigación bibliográfica citada

Korbitz y otros (2003) revisan el panorama mundial en materia de políticas de biocombustibles, valiéndose de cuestionarios enviados a los agentes responsables de la formulación de políticas para el sector energético. Los autores notan que, en todos los países, la principal motivación en la adopción de biocombustibles es la reducción de emisiones de gases. El incentivo ha promovido principalmente la mezcla en proporciones reducidas con el diésel. En los países de América se puede encontrar, además de la idealización de subsidios y tributos, incentivos para instalación de plantas de procesamiento, de forma evidente en los Estados Unidos, Brasil y Argentina. Las actividades de investigación y desarrollo han sido aplicadas en todos los países importantes en la producción de biocombustibles, principalmente en Alemania, Francia, Brasil, Estados Unidos y Australia. 
Políticas para relaciones contractuales en las cadenas de biocombustibles: construyendo puentes entre instituciones

Ragauskas (2006) infiere que la producción de biocombustibles en un contexto de cambio de paradigma en la industria genera nuevas posibilidades de innovación. Mientras tanto, nuevas tecnologías aún necesitarían un esfuerzo adicional de investigación y desarrollo para ampliar su actuación en la producción de materiales en general.

Van Thuijl y Deurwaarder (2006) califican a los países del Este europeo como atrasados en cuanto a políticas de implementación de biocombustibles, especialmente Polonia, donde la inestabilidad de reglas para el sector ocasiona un desestímulo, en la medida en que decisiones de inversión de mediano y largo plazo son perjudicadas. En los casos de Holanda, Reino Unido y Malta, los autores entienden que apenas una política de largo plazo tornaría viable el uso de biocombustibles en estos países, pues políticas públicas de subsidios y exenciones tarifarias son antieconómicas. De modo general, aún así se aprecia dentro del contexto europeo un mercado bastante cerrado frente a las oportunidades que el mercado ofrece, además de ausencia de coordinación entre los agentes envueltos en la cadena.

\section{PROBLEMAS DE COORDINACIÓN EN LAS CADENAS PRODUCTIVAS DE BIOCOMBUSTIBLES}

Por la descripción realizada previamente, se evidencia que existen especificidades en las cadenas de biocombustibles, ya que todas las materias primas usadas hasta el momento poseen usos alternativos en la industria de alimentos y no pueden ser sustituidas tan fácilmente a corto plazo.

Las cadenas productivas de soya, maíz y girasol son consolidadas y lideradas por grandes empresas con estrategias globales de actuación. Las mismas han sido incentivadas a invertir en biodiésel y alcohol de maíz, pero el alza de precios internacional de estos productos afecta otras cadenas e incentiva el uso más tradicional. De esta manera, la producción de biocombustibles se torna en la causa de los aumentos de precios, lo que afecta su producción. Otros mecanismos de coordinación deben ser creados para garantizar la producción y estabilidad. Los agentes deben ser incentivados a invertir en energía.

La cadena productiva del alcohol-combustible es fuertemente influenciada por el precio internacional del azúcar, el cual generó impactos importantes en la producción del pasado y contribuyó para el desabastecimiento del mercado con la existencia de cuotas de producción y precios tabulados.

Otras cadenas productivas se están consolidando. Las formas de gobernabilidad no están claras y aún falta transparencia. La mamona ${ }^{5}$ es vista como la mejor fuente

5 Semilla de higuerilla o ricino. 
de materia prima para el biodiésel en Brasil, pero la cadena aún no esta estructurada, los intereses son dispersos y no existe consenso. Otro factor importante es que el aceite de mamona tiene un uso noble en la industria de aviación y en otros sectores metalmecánicos, con precios elevados y sin sustitutos próximos y a corto plazo.

Se puede mencionar también la palma, que ha sido vista como otra fuente potencial de materia prima de biodiésel en Brasil y Asia. En esta última, el avance del cultivo está causando un gran impacto ambiental por la tala de bosques para su cultivo. Este producto también presenta un uso noble y corriente: el la industria de alimentos, margarinas, aceites especiales, alimentos industriales, y se debe buscar la consolidación de su uso como combustible en el futuro.

En Colombia, la industria de palma se encuentra en pleno proceso de desarrollo como opción para sustituir los combustibles fósiles. Mesa (2007) apunta que la normativa impuso a partir de enero de 2008 la condición de incorporar en los combustibles $5 \%$ de biodiésel, lo que hizo que la producción de aceite de palma aumentara en un $133 \%$ entre 2007 y 2008. La estrategia aplicada en Colombia para incentivar el cultivo de palma y la producción de aceite de palma se ha centrado principalmente en la exención de impuestos. Las principales estrategias utilizadas en Colombia para promover el desarrollo de la producción se pueden clasificar en tres grupos: creación de un marco legal, creación de un marco económico y creación de un marco técnico. El primer grupo, que es esencial en la fase de implantación, se centra en acciones relacionadas con la normativa y la reglamentación. En el segundo grupo, que es muy relevante en la fase de consolidación del mercado, se centra en acciones relacionadas con el riesgo, la formación de precios y canales de comercialización. Finalmente, el tercer grupo, que es fundamental para el ejercicio de la compensación ambiental, se centra en acciones relacionadas con la viabilidad técnica y la infraestructura.

En Parsons (2007) se confirma la necesidad de integración de diversas áreas del conocimiento para el éxito de la política pública. Los niveles de gobernanza son definidos por los potenciales sociales y regionales disponibles desde la perspectiva de resultados. Gran parte de los resultados se pueden atribuir no solamente a los beneficiarios directos, puesto que las repercusiones en tejido social y en otros sectores son seguras y afectan el desarrollo económico en todas sus dimensiones.

En esta discusión se presentan los puntos de vista de Salazar (2009), donde la evaluación de las políticas públicas se condiciona a múltiples dimensiones en las que se confrontan intereses de la sociedad, del Gobierno y de los sectores económicos, de forma que la intensidad de integración de políticas públicas en diversos actores contribuye a determinar los efectos y la amplitud de resultados. Las políticas públicas para biocombustibles tienen ese poder de generar y distribuir resultados 
Políticas para relaciones contractuales en las cadenas de biocombustibles: construyendo puentes entre instituciones

en diversos sectores sociales: desde los campesinos hasta el Gobierno, pasando por la población urbana.

Hasta este punto se han discutido los impactos de la falta de consolidación de las relaciones entre la agroindustria y la agricultura. Pero, existen problemas más importantes en las relaciones entre la agroindustria y los distribuidores de combustibles. Estudiando el caso de Brasil, aún existen conflictos en la fijación de precios, formación de stocks, garantías de la calidad, entre otros aspectos. En otros países la distribución regionalizada de biocombustibles causa trastornos a los consumidores, que no pueden viajar a ciertas regiones.

En el caso de países en desarrollo, se deben citar también las cuestiones laborales, pues las relaciones entre los agricultores y los trabajadores rurales están marcadas por conflictos, y muchos países no tienen una legislación y fiscalización efectivas para impedir los abusos.

En conclusión, a partir de lo que se ha discutido hasta el momento, las políticas de biocombustibles buscan la solución de problemas del abastecimiento a corto plazo, y dejan de lado importantes lagunas institucionales y de coordinación. Esto puede contribuir a la resolución de los problemas de abastecimiento a corto plazo, pero a su vez contribuye a generar, a largo plazo, importantes efectos negativos de carácter social y económico.

\section{EXPERIENCIAS DE COORDINACIÓN DE LAS CADENAS Y SUS POSIBLES LECCIONES EN BRASIL}

En esta sección se revisan algunas experiencias de coordinación entre los eslabones de la cadena de producción. La experiencia brasileña con la producción de alcohol comenzó en el inicio del siglo XX. Durante una buena parte de este siglo, el sector de producción de caña fue planeado por el Estado a través del Instituto del Azúcar y Alcohol (IAA). El mismo puso en práctica una serie de políticas que contribuyeron a disminuir y disimular los conflictos entre los proveedores de materia prima y la agroindustria, y de estas con los comerciantes del azúcar y del alcohol. Con la falta de reglamentación para el sector en los años 1990, estos conflictos resurgieron nuevamente.

Uno de los hechos más importantes de este período fue la creación de grupos de comercialización de alcohol y azúcar para ajustar o equilibrar el poder de negociación de los agentes. Después del fracaso de BBA (Bolsa Brasileira de Álcool) y de Brasil Álcool, los empresarios del sector se reunieron en grupos para reducir los costos y vender alcohol a precios más competitivos. 
Azevedo (2000) señala que esta estrategia mejoró el poder de negociación de los productores rurales y agroindustriales frente a las grandes industrias de alimentos y al por menor. El autor resalta que en muchos casos las asociaciones pueden tener vida corta, pues la coordinación ex-post de las acciones de los agentes individuales requiere fuertes incentivos para el cumplimiento de las reglas y genera costos de transacción elevados. Según Vian (2003), los grupos de comercialización y cooperativas solo obtienen el éxito cuando los beneficios de la acción coordinada son reconocidos por todos.

De acuerdo con Marques (2012), una de las primeras asociaciones de productores fue Crystalsev, un pool de compras y comercialización formado por Santa Elisa, Vale do Rosario, MB, Moema, Jardest, Pioneiros, Mandu, Cevasa y, recientemente Equipav. Este grupo tuvo éxito por causa de los puntos en común de las empresas, de su tamaño, mix de productos y de la administración profesional. Empresas como Santa Elisa y Vale do Rosario tienen un pasado de cooperación y de sociedad. Ellas son accionistas de MB y de Moema.

Para Vian (2003), la cooperación dio resultados inesperados: Vale do Rosario se fusiono con Jardest, lo que fortaleció las empresas y el grupo de comercialización, Crystalev es un ejemplo de cómo los puntos en común son importantes en la hora de decidir por la cooperación y por las fusiones, pues los conflictos son minimizados.

Esta experiencia con Crystalev capacitó a su principal director, João Carlos Figuereido Ferraz, para ser uno de los articuladores de la fundación BBA y de Brasil Álcool, tornándose uno de los actores principales del sector en la actualidad.

BBA no tuvo una larga vida, aunque consiguió cumplir la meta de elevar los precios del alcohol. La primera disidencia de BBA fue Copersucar, que dejó la empresa y cerró contratos a largo plazo y con garantía de exclusividad con una gran distribuidora, pues tuvo mayor incentivo para actuar independientemente que en bloque. Según Vian (2003), de esta manera, las partes evitaron problemas de comercialización y grandes variaciones de precios.

De acuerdo con Aluízio Nunes ${ }^{6}$, Copersucar tiene por objetivo garantizar los intereses comerciales de sus asociados, y por esa razón no podía amarrarse a una asociación en la que decenas de empresas tenían poder de voto. Por otro lado, Copersucar tiene montada una estructura propia de comercialización y no tiene por

Información obtenida en entrevista realizadas por los autores. 
Políticas para relaciones contractuales en las cadenas de biocombustibles: construyendo puentes entre instituciones

qué pagar comisiones para que terceros vendan el alcohol de sus asociadas. Para Nunes, Copesucar participo de BBA y de Brasil Álcool porque el momento era de crisis y el sector necesitaba mantenerse unido. Pero la propia cooperativa, según Vian (2003), mostró que en organizaciones en las que muchas empresas participan, casi nunca se llega a un acuerdo. Lo ideal es la formación de pequeños grupos con sinergias e intereses en común.

Esta opinión parece ser compartida por otros ejecutivos y dirigentes del sector, pues después de la disolución de BBA surgieron grupos menores, que buscaban sinergias e intereses comunes. Marques (2012) plantea que en todos los casos se hizo evidente que cada grupo tiene su propio liderazgo y que los participantes tenían intereses y orígenes comunes.

De la misma forma que con BBA, los pools de fábricas fortalecen el sector para negociar con las distribuidoras que, históricamente, consiguieron imponer precios bajos para las fábricas en acuerdos aislados. Los grupos pueden disciplinar el sector a través de la cooperación, fortalecen los mercados con oferta suficiente del producto, sin perjudicar al consumidor. Así, el sector puede contribuir para la mejora de su imagen frente a la opinión pública.

La formación de grupos de comercialización demuestra la madurez y el conocimiento generado por la crisis de 1999. El complejo de cultivadores de caña ha logrado finalmente construir una autogestión de las actividades productivas y políticas. Según Marques (2012), constatar que no es posible construir un consenso entre las decenas de fábricas del centro-sur es un aporte importante, pues muestra que el sector está dispuesto a discutir los problemas organizacionales y comerciales en grupos menores, donde los intereses comunes son mayores, incentivan la cooperación y disminuyen la competencia entre los agentes.

Por el lado político, UNICA ha logrado obtener éxito al conciliar los intereses de las fábricas paulistas, pero sin mencionar los hechos comerciales que quedan a cargo de las unidades individuales y de los grupos de comercialización.

Uno de los principales grupos formados con la disolución de BBA fue la Sociedade Comercializadora de Álcool (SCA) un pool formado por 31 fábricas de la región centro-sur (ver cuadro 2) y que es administrado por el mismo equipo que integró BBA (Bolsa Brasileira de Álcool). Copesucar continua con la mayor parte, 25 \%, y SOL, liderada por el grupo José Pessoa con 11 \% (Canaweb). 
Cuadro 2. Estructura de los grupos de comercialización de Alcohol.

\begin{tabular}{|c|c|}
\hline Grupo & Plantas de producción afiliadas \\
\hline SCA & $\begin{array}{l}\text { Cosan, Crystalsev, Da Barra, Dois Córregos Goiasa, Jalles Machado, } \\
\text { Monte Alegre, Albertina, Bazan, Centralcool, Cocal, Colorado, Paraíso, } \\
\text { Santa Cândida, Santa Fé, Maracaí, Nova América. }\end{array}$ \\
\hline SOL & $\begin{array}{l}\text { Usinas Santa Fé, Santa Helena, Sonora Estância, Debrasa e Santa Olinda, } \\
\text { Alvorada do Bebedouro, Sanagro-MG, Santo Antônio, benalcool, Gasa, } \\
\text { Sanagro -SP, Unialco y Univalem, Sanagro-SE y Una }\end{array}$ \\
\hline Bioagência & $\begin{array}{l}\text { Grupo João Lyra, Moreno, Bertolo E Cia Ltda., Delta/Volta Grande, Desti- } \\
\text { laria A.Ruette, Destilaria Alta Floresta, Destilaria Della Coletta, Destilaria } \\
\text { Ferrari, Destilaria Pitangueiras, Destilaria Santa Inês, Floralcool, Irmãos } \\
\text { Malosso, Iturama, Usina Alta Mogiana, Usina Guairá, Usina Nardini, Usina } \\
\text { Santa Isabel, Usina Zanin y Virálcool. }\end{array}$ \\
\hline Copersucar & $\begin{array}{l}\text { Barra Grande, Batatais, Bela Vista-Pontal, Bom Retiro, Buriti, Catanduva, } \\
\text { Da Pedra, Furlan, Ibirá, Ipiranga Iracema, Modelo, N.S.Aparecida-Itapira, } \\
\text { Paredão, Porto Feliz, Quatá, Rafard, Santa Adélia, Santa Cruz-Ab, Santa } \\
\text { Cruz-Capivari, Santa Lucia, Santa Luiza, Santa Maria-Cerquilho, Santa } \\
\text { Rosa, Santa Teresinha, Santo Alexandre, Santo Antonio-Piracicaba, } \\
\text { Santo Antonio-Sertãozinho, São Carlos,.São Francisco-Sertãozinho, } \\
\text { São João-Araras, São José-Macatuba, São José- Rp, São José Da Estiva, } \\
\text { São Luiz-Ourinhos, São Luiz-Pirassununga, São Manoel, São Martinho, } \\
\text { Santa Philomena, Santa Luiza-Jaboticabal y São Gregório. }\end{array}$ \\
\hline $\begin{array}{l}\text { Pool de } \\
\text { Exportación }\end{array}$ & Lasa, Alcon, Cridasa, Albesa y Serra dos Aymorés. \\
\hline
\end{tabular}

Fuente: elaborado por los autores a partir de datos primarios obtenidos de las empresas y Unica

Los asociados de SCA se reúnen una vez por semana para discutir la política de comercialización, ventas realizadas y estrategias de precio. Según el señor Jacir, corredor ejecutivo, el tamaño y la tradición de los asociados facilitan el consenso sobre la política de comercialización; no existen voces disonantes que pidan acciones oportunistas.

SOL, dirigido por el grupo J. Pessoa, también ha tenido buenos resultados. El pool es formado por 18 fábricas (ver cuadro 2) de Mato Grosso do Sul, Minas Gerais, São Paulo, Sergipe y Paraiba. Otras unidades de los estados de Goias y Minas Gerais en la región centro-sur y Alagoas, en el noroeste, están negociando su participación.

$7 \quad$ Información obtenida en entrevista realizada por los autores. 
Políticas para relaciones contractuales en las cadenas de biocombustibles: construyendo puentes entre instituciones

Un factor observado por el director de SOL, José Pessoa de Queiroz Bisneto ${ }^{8}$, es que, independiente del precio del producto, el grupo puede discutir sobre cuándo destinar o no su producción para el alcohol con fines de evitar el desabastecimiento. Según él, en la cosecha de 2000/2001, SOL destinó prácticamente toda su producción al alcohol por una cuestión de responsabilidad con los consumidores del combustible renovable y con el propósito de mejora de la imagen del sector frente al consumidor. Él declaró también que los bloques de comercialización surgieron para sustituir a BBA, ya que son más eficientes, buscan la identificación mutua, mientras que BBA concentraba un gran número de empresas con características diferentes (Marques, 2012).

Actuando de forma homogénea y con personalidad propia, los bloques de comercialización avanzan hacia otros Estados, como es el caso de Paraná, que tiene CPA (Central Paraná de Álcool). Según Paulo Zanetti ${ }^{9}$, director ejecutivo de la extinta Cepaal (Coligação das Entidades Produtoras de Açucar e Álcool), en los próximos años los grupos de productores comercializarán de forma directa y profesional, no solo el alcohol, sino también los otros derivados de la caña, como por ejemplo, la energía eléctrica.

Es importante que el sector busque perfeccionar la comercialización y la logística, con el uso de más instrumentos de mercado. Los bloques deben atraer mayor financiamiento y crédito, que refleje mayor seguridad, estabilidad y rentabilidad para toda la cadena productiva de alcohol y de azúcar y evite crisis de coordinación como la de 1999.

Hasta este punto puede concluirse que, los grupos configuran verdaderos Campos Organizacionales, pues congregan empresas con estrategias y visiones de mundo semejantes y con estructuras productivas y administrativas cercanas. De acuerdo con Vian (2003), ellas pueden cooperar y competir, ya que se conocen y pueden controlar el oportunismo.

Cuatro destilerías de Espirito Santo y una de Minas Gerais (ver cuadro 2) exportan alcohol como alternativa para equilibrar el mercado de alcohol de azúcar, principalmente en la época de cosecha o recolección. Es en este período que fábricas de Río de Janeiro y de Minas Gerais entran en Espirito Santo y fuerzan la disminución del precio del producto, lo que descapitaliza las empresas locales en un frágil momento, de costeo del plantío y cosecha. Según Vian (2003), se espera que la incursión de las empresas locales en la exportación se convierta en alivio para la presión sobre los precios.

Información obtenida en entrevista realizada por los autores.

Información obtenida en entrevista realizada por los autores. 
Después del primer embarque experimental, ese grupo se prepara para la segunda venta a Europa y Asia. Serán exportados 15 millones de litros de alcohol hidratado, utilizado como insumo para industrias químicas, lo que resultará en una cuantía de US\$ 3.6 millones.

Aunque hoy la idea básica de pool sea la de transformar el mercado externo en alternativa para mantener la facturación, sin las tradicionales oscilaciones, en el futuro los empresarios esperan que las exportaciones puedan tener un peso mayor en las estrategias y cuantías de las destilerías. Las expectativas actuales son que el mercado europeo decida sobre la adición de alcohol a la gasolina, como ocurre en Brasil. Actualmente, la mezcla en Europa es hecha con alcohol de remolacha y maíz, el que cuesta el triple que el biocombustible brasileño.

El grupo comenzó a formarse a inicios del 2001 y está abierto a nuevas adiciones. La fábrica Paineiras, localizada en la región sur, admitió su interés en el negocio, pero la dirección consideró conveniente esperar un poco. Las exigencias para entrar al grupo son: credibilidad, garantía de producto y voluntad de actuar en conjunto con otras empresas.

En el proceso de cambio institucional del Complejo cultivador de caña Brasileño, una categoría que ha sufrido grandes impactos es la de proveedores de caña en el Estado de São Paulo, y también en otros Estados, pues se sabe poco sobre las características y necesidades de estos agentes en términos de contratación de mano de obra, financiamiento, entre otros aspectos relevantes, como la relación contractual con las fábricas.

En ese sentido, el sector aún tiene resquicios de la fase de regulación estatal, cuando las relaciones eran orientadas por IAA y cuando era difícil controlar el cumplimiento del porcentaje de caña exigido a los proveedores. Actualmente con el libre mercado y las nuevas formas de coordinación, el Estado debe preocuparse apenas por la determinación de un marco legal para los contratos, y dejar que las partes se entiendan. Volver a crear leyes o normas que obligue la compra de caña de proveedores sería un retroceso en el aprendizaje conseguido e interferiría con las estrategias de los agentes.

De esta forma, el Estado debe adoptar políticas de apoyo a la investigación para que puedan entenderse mejor las necesidades y las orientaciones actuales para una actividad ambientalmente correcta y socialmente sustentable. Es urgente el aumento de investigaciones en estos temas. Esto puede ayudar a los productores a mejorar los costos de producción, sistemas de investigación de precios por medio de Consecana, entre otros aspectos productivos. 
Políticas para relaciones contractuales en las cadenas de biocombustibles: construyendo puentes entre instituciones

En cuanto al sistema Consecana, fue una respuesta eficiente del sector al proceso de falta de reglamentación y aún esta en proceso de maduración y pasa por revisión periódica. Según Belik (2010), este mecanismo no debe sufrir interferencias del Estado, pues puede generar un retroceso e interferir en las conductas estratégicas de las empresas.

La estrategia de profundización ha afectado a los pequeños productores, pero ellos pueden tener acceso a nuevas tecnologías a través de asociaciones y cooperativas y del proceso de tercerización de las actividades agrícolas. Por otro lado, puede incentivarse a los pequeños a adoptar tecnologías para actuar en otros mercados derivados de la caña, como aguardiente, panela, melado y otros tipos de alimentos y productos. Los estudios en este campo son poco explorados en Brasil y representan una alternativa de renta importante para estos agentes.

La recién creada estructura de producción de Biodiésel también presenta problemas de coordinación entre varios eslabones de la cadena productiva, desde la relación con las instituciones financieras de producción hasta las de abastecimiento de materia prima. Las subastas para adquisición de producto fueron exitosas, pero muchas empresas no están cumpliendo los contratos con Petrobrás por no tener materia prima para producir o por estar produciendo aceite para la industria de alimentos.

El mercado de alcohol-combustible en Brasil ha tenido variaciones de precios más significativas y comentadas porque es un producto que tiene más impacto en los índices de inflación y en la estructura productiva. La relación entre distribuidores de combustibles y fábricas aún no es de las más estables, aún con la existencia de los grupos de comercialización de alcohol. Aún es necesario madurar más en este sentido. Así se hace necesaria la adopción de una política de stocks reguladores que deberían ser negociados entre los diversos eslabones de la cadena y del Estado, que darían mayor estabilidad a los precios, ya que la política de alteración de mezcla para gasolina no resuelve el problema.

Una política de stocks beneficiaría mucho al consumidor y podría dar una orientación mejor del comportamiento de precios, a la vez que evita la toma de decisiones equívocas en momentos de euforia.

Existen algunas controversias sobre cuál debe ser el volumen en stock. Algunos representantes del sector dicen que debe ser de 3 billones de litros. Aún así existen muchas divergencias sobre quién costeará financieramente esa acción. Este costo debería ser distribuido entre los diversos eslabones de la cadena y no pagado en su totalidad por el consumidor final a través de precios elevados en los períodos que transcurren entre una cosecha (recolección) y otra. 
Otra forma de evitar los impactos de los períodos entre cosechas es a través de investigación de nuevas variedades y materias primas, para que el funcionamiento de las fábricas sea mayor a lo largo del año. Muchas ya están funcionando hasta por mueve meses. Así, se evitaría el impacto de la parada de producción, característica de un producto agroindustrial.

Se ha discutido bastante sobre el desarrollo del futuro mercado del alcohol, pero este no ha crecido en Brasil, por causa del desinterés de los agentes, poca información sobre mecanismos de mercado, tradicionalismo en las fábricas y falta de recursos para cubrir las márgenes de garantía; las intervenciones en estos temas pueden contribuir a resolver el problema de escasez en la entre-cosecha y por las características del sector productivo, principalmente la integración vertical hacia atrás de las fábricas.

Se debe destacar que todas las iniciativas descritas se han desarrollado por iniciativa del sector privado. El Estado debería usar estas iniciativas como punto de partida para la implementación de políticas públicas de estabilización de precios.

El mercado de capitales comienza a ser identificado como una fuente de financiamiento de bajo costo para el sector. El lanzamiento de acciones en la bolsa por parte de grupos importantes y la adopción de mecanismos de Gobierno para garantizar la transparencia son inéditos y deben crecer en el futuro. La continuidad de la política económica deberá incentivar esta estrategia de financiamiento.

Por otro lado, las estrategias adoptadas por los grandes grupos a través de prácticas de coordinación vertical, sustentabilidad ambiental, responsabilidad social y competitividad pasan a ser copiadas y comienzan a convertirse en patrón de conducta en ese segmento productivo. Pero se hace necesaria la definición de estándares de calidad socioambiental, de productos y procesos y sus certificaciones. Para el consumidor final es difícil saber si el estándar de diferenciación es el que realmente definieron las empresas. De este modo, el Estado debe incentivar la discusión de estándares de calidad y de certificación para que la estrategia de diferenciación se torne más atractiva.

Mecanismos de calidad y certificación y de estabilización de precios deberán disminuir la adulteración del producto y permitir que los consumidores den más importancia a los estándares de calidad que a los precios.

El análisis anterior evidencia que las instituciones, es decir, el Estado y las políticas públicas, la cultura organizacional, la estructura social y la forma de organización de los agentes económicos y sociales influyen de manera significativa en la 
Políticas para relaciones contractuales en las cadenas de biocombustibles: construyendo puentes entre instituciones

formulación de estrategias empresariales y en sus cambios. Conforme fue descrito, la política de intervención estatal en el cultivo de caña nacional fue responsable por una estructura atrasada y de baja competitividad que se mantuvo en los últimos cincuenta años. Los cambios de política y de ambiente institucional obligan a las empresas a adoptar estrategias diferenciadas de las anteriores, para buscar la competitividad nacional e internacional.

Antes, las estrategias individuales estaban subordinadas a una regulación general establecida por el Estado interventor. Lo que es nuevo es el surgimiento de nuevas estrategias individuales basadas en la diferenciación del producto, diversificación productiva y especialización. Estos movimientos son indicativos de las dificultades para reestablecer el antiguo consenso en relación con las condiciones de producción. Si existe un movimiento común a todos los cultivadores de caña del centro-Sur de Bahía, este es el de la concentración/centralización de capitales, que nuevamente se ha hecho presente.

Con todo, futuras investigaciones sobre los complejos regionales y la adopción del análisis institucional, así como una reflexión más profunda de las estrategias competitivas que son adoptadas son necesarias para la adopción de políticas públicas adecuadas.

\section{CONSIDERACIONES FINALES SOBRE LAS DIRECTRICES DE POLÍTICAS PARA MEJORAS EN LAS RELACIONES CONTRACTUALES}

Como se ha enfatizado desde el inicio del texto, el propósito de este trabajo es revisar las políticas utilizadas para mejorar e incentivar el desarrollo del sector de biocombustibles, y mejorar las relaciones contractuales en la cadena de biocombustibles que son marcadas por especificidades de los productos, procesos y características regionales e institucionales.

La principal lección del estudio del caso brasileño es que el desarrollo y la consolidación de las relaciones son lentos y están expuestos a múltiples dificultades. A partir de esta retrospectiva se pueden nombrar las siguientes directrices de políticas con el propósito de que sirvan para disminuir los conflictos entre los eslabones de las cadenas.

Las políticas deben tomar en cuenta las condiciones locales y regionales de producción, los intereses ya establecidos y el conocimiento ya adquirido:

- Se debe tomar en cuenta que los biocombustibles tienen diversos usos, y la producción y el consumo local pueden contribuir para la reducción del con 
flicto, pues el espacio de interacción entre los agentes ya existe y puede ser consolidado;

- El proceso de certificación social y ambiental es una buena forma de incentivar las relaciones duraderas y con pocos conflictos, pues los costos de certificación inducen a la cooperación.

- Debe incentivarse a la diversificación productiva en todos los eslabones de la cadena, pues esto disminuye la dependencia mutua y posibilita menores conflictos, además de incentivar la cooperación;

- Inducir a la mejora tecnológica es un factor importante para la reducción de conflictos, pues lleva a comportamientos cooperativos entre los productores, proveedores, unidades de investigación, entre otros;

- Definición del papel de los biocombustibles en la matriz energética de los países productores, que evita los problemas a corto plazo e induce a la cooperación y alianzas de largo plazo.

Esta investigación sobre el caso brasileño es importante y nos hace reflexionar sobre muchas cuestiones. Pero, se debe invertir en investigaciones comparativas entre los países productores de biocombustibles y en la divulgación de sus problemas y acciones. Esto se puede hacer con la construcción de una red de cooperación entre los investigadores de América Latina y Europa.

\section{BIBLIOGRAFÍA}

Azevedo, P. F. (2000). Nova economia institucional: referencial geral e aplicações para a agricultura. En: Revista Agricultura em São Paulo, Vol. 47, N. . 1, pp. 33-52.

Bathia, R. (1985). Energy and Agriculture in Developing Countries. En: Energy Policy, Vol. 13, N. 4, pp. 330-334.

Belik, Walter (2010). Desenvolvimento Rural e Segurança Alimentar: Contribuições Teóricas para o Desenho e Avaliação de Políticas Públicas. Tese para o Concurso de Professor Titular em Economia Agrícola apresentada ao Instituto de Economia da Unicamp, Campinas (mimeo)

Bernard, F y Prieur, A. (2007). Biofuel market and carbon modeling to analyse French biofuel policy. En: Energy Policy, Vol. 35, N. 12, pp. 5991-6002.

Bernetti, I.; Fagarazzi, C. y Frantini, R. (2004). A methodology to analyse the potential development of biomass energy sector: an application in Toscany. En: Forest Policy and Economics, Vol. 6, pp. 415-432. 
Políticas para relaciones contractuales en las cadenas de biocombustibles: construyendo puentes entre instituciones

Brannlund, R. y Kristrom, B. (2001). Too hot to handle? Benefits and costs of stimulating the use of biofuels in the Swedish heating sector. En: Resource and Energy Economics, Vol. 23, N. ${ }^{\circ}$, pp. $343-358$.

CMDE -Comissão mundial sobre meio ambiente e desenvolvimento (1991). Nosso Futuro Comum. $2^{a}$ Ed., Rio de Janeiro: Editora da Fundação Getúlio Vargas, 430p.

Costa, R. C. (2004). Potential for producing biofuel in Amazon deforested areas. Biomass and Bioenergy, Vol. 26, N. ${ }^{\circ}$, pp. 405-415.

Dautzenberg, K. y Honf, J. (2008). Biofuel chain development in Germany: Organization, opportunities and challenges. En: Energy Policy, Vol. 36, N. 1, pp. 485-489.

Demirbas, A. y Dermibas, I. (2007). Importance of rural bioenergy for developing countries. En: Energy Conservation and Management, Vol. 48, N. ${ }^{\circ}$ 8, pp. 2386-2398.

Dishington, J. M. (2007). Biodiésel de palma: una realidad en Colombia [En línea] Fedepalma $<$ http://portal.fedepalma.org//biodiesel__ col.htm> Consultado: Enero de 2012.

Downing, M.; Volk, T. A.; y Schimidt, D. A. (2005). Development of a new generation cooperatives in agriculture for renewable energy research, development, and demonstration projects. En: Biomass and Bioenergy, Vol. 28, N. ${ }^{\circ}$, pp. 425-434.

Faaij, A. P. C. (2006). Bio-energy in Europe: changing technology choices. Energy Policy, Vol. 34, N. 3 , pp. 322-342.

FAO - Organização das Nações Unidas pra Agricultura e Alimentação (2007). First FAO Technical Consultation on BioEnergy and Food Security. Roma: FAO.

Forge, F. (2007). Biofuels - An Energy, Environmental or Agricultural Policy? Ottawa: Library of Parliament, Parliamentary Information and Research Service.

Gowen, M. (1989). Biofuels versus Fossil Fuels in developing countries: How green is the pasture? En: Energy Policy, Vol. 17, N. ${ }^{\circ}$ 5, 1989, pp. 455-470.

Hagerdal, B. H.; Galbe, M.; Grauslund, M. F. G.; Liden, G. y Zacchi, G. (2006). Bio-ethanol - The Fuel of Tomorrow From The Residues of Today. En: Trends in Biotechnology, Vol. 24, N. ${ }^{\circ}$ 12, pp. 549-56.

Hamelinck, C. N.; Suurs, R. A. A. y Faaij, A. P. C. (2005). International Bioenergy Transport Costs and Energy Balance. En: Biomass and Bioenergy, Vol. 29, N. ${ }^{\circ} 2,2005$, pp. 114-134.

Heilbroner, Robert. (1996). A História do Pensamento Econômico. São Paulo: Nova Cultural, 319 p. Hobsbawm, Eric. (1995). A Era dos Extremos: Uma breve história do século XX. São Paulo, Companhia das Letras, 598 p.

Semestre Económico, volumen 16, №. 33, pp. 15-44 • ISSN 0120-6346, enero-junio de 2013, Medellín, Colombia 
Islam, A. K. M. S.; Islam, M. y Rahman, T. (2006). Effective Renewable Energy Activities in Bangladesh. En: Renewable Energy, Vol. 31, N. 5, pp. 677-688.

Jull, C.; Redondo, P. C.; Mosoti, V. y Vapnek, J. (2007). An Overview of National Legal and Policy Frameworks for Bioenergy Production, Promotion and Use. Roma, [En linea] FAO. <http:// www.globalbioenergy.org/uploads/media/0709_FAO_ legal _ paper_online _ _ _ RECENT_TRENDS _ IN _ THE_LAW_AND_POLICY _ OF_BIOENERGY _ PRODUC TION__ PROMOTION_AND_USE_ 01.pdf $>$. Consultado: enero de 2012.

Klass, D. L. (1995). Biomass Energy in North American policies. En: Energy Policy, Vol. 21, N. 12, pp. $1035-1048$.

Korbiz, W.; Friedrich, S.; Waginger, E. y Worgetter, M. (2003). Worldwide Review on Biodiesel Production. En: IEA Bioenergy Task 39, Subtask "Biodiesel", Viena: Austrian Biofuels Institute.

Kruse, J.; Westhoff, P.; Meyer, S. y Thompson, W. (2007). Economic Impacts of Non Extending Biofuel Subsidies. En: The Journal of Agrobiotechnology Management $\mathcal{E}$ Economics, Vol. 10, N. 2 , pp. 94-103.

Lang, X.; Dalai, A. K.; Bakhshi, N. N.; Reaney, M. J. y Hertz, P. B. (2001). Preparation and Characterization of Bio-Diesels From Various Bio-Oils. En: Bioresource Technology, Vol. 80, N. ${ }^{\circ}$ 1, pp. 53-62.

Leite, A. D. (1997). A energia do Brasil. Rio de Janeiro: Ed. Nova Fronteira, 500p.

Lewandowski, I.; Weger, J.; Van Hooijdoonk, A.; Havlickova, K.; Van Dam, J. y Faaij, A. C. P. (2006). The potential biomass for Energy Producing in the Czech Republic. En: Biomass and Bioenergy, Vol. 30, pp. 405-421.

Macedo, I. C. (2007). Situação Atual e Perspectivas do Etanol. En: Estudos Avançados, Vol. 21, N. ${ }^{\circ}$ 9, pp. 157-165.

Marin, F. R.; Assad, E. D.; Barbarisi, B. F.; Pilau, F. G. y Pinto, H. S. (2007). Efeitos das Mudanças Climáticas sobre a Aptidão da Cana-de-Açúcar no Estado de São Paulo. En: XV Congresso Brasileiro de Agrometeorologia -CBAGRO, 2007, Aracaju. XV Congresso Brasileiro de Agrometeorologia - CBAGRO. Campinas: SBAGRO, 2007. Vol. 1. pp. 12-18.

Martin, Jean Marie. (1989). A Economia Mundial da Energia. São Paulo: Ed. Unesp, 135 p..

Mathews, J. A. (2007). Biofuels: A Biopact Between North and South Could Achieve. En: Energy Policy, Vol. 35, N. ${ }^{\circ}$, pp. 3550-3570.

Marques, D S P.; Paulillo, L. F. O. E. y Vian, C. E. F. (2012). Grupos de comercialização de etanol e governança em rede no Brasil. En: Gestão \& Produção (UFSCAR. Impresso), Vol. 19, pp. 832-847. 
Políticas para relaciones contractuales en las cadenas de biocombustibles: construyendo puentes entre instituciones

Ometto, A. R.; Ramos, P. A. R. y Lombardi, G. (2007). The Benefits of a Brazilian Agro-Industrial Symbiosis System and the Strategies to Make it Happen. En: Journal of Cleaner Production, Vol. 15, N. ${ }^{\circ} 13-14$, pp. 1253-1258.

Oztertuk, H. H. y Bascetincelik, A. (2006). Energy Exploitation of Agricultural Biomass Potential in Turkey. En: Energy Exploration E Exploitation, Vol. 24, N. ${ }^{\circ} 1-2$, pp. 95-111.

Parsons, W. (2007). Public policy: an introduction to the theory and practice of policy analysis. Edward Elgar, Cheltenham, 2003. Traducción: Políticas públicas: una introducción a la teoría y la práctica del análisis de políticas públicas. Miño y Dávila, Buenos Aires.

Pippo W., A.; Garzone, P. y Cornacchia, G. (2007). Agro-Industry Residues Disposal: The Trends of Their Conversion Into Energy Carriers in Cuba. En: Waste Management, Vol. 27, N. 7 , pp. 869-885.

Ragauskas, A. J.; Williams, C. K.; Davison, B. H.; Britovsek, G.; Cairney, J.; Eckert, C. A.; Frederick Jr., W. J.; Hallett, J. P.; Leak, D. J.; Liotta, C. L.; Mielenz, J. R.; Murphy, R.; Templer, R. y Tschaplinski, T. (2006). The Path Forward for Biofuels and Biomaterials. En: Science, Vol. 311, N. ${ }^{\circ} 5760$, pp. 484-489.

Rozakis, S.; Sourie, J. C. y Vanderpooten, D. (2001). Integrated Micro-Economic Modelling to Support Public Decision Making: The Case of Liquid Bio-fuels in France. En: Biomass and Bioenergy, Vol. 20, pp. 385-398.

Rozakis, S.; Sourie, J. C. (1998). Energy from agricultural biomass and effects on rural income in Thessalia, Greece in Zazueta, F. S.; Xin, J. S. (orgs.) En: Computers in Agriculture, pp. 300-305.

Russi, D. (2008). An Integrated Assessment of a Large-Scale Biodiesel Production in Italy: Killing Several Birds With One Stone? En: Energy Policy, Vol. 36, N. ${ }^{\circ} 3$, pp. 1169-1180.

Salazar Vargas, C. (2009). La evaluación y el análisis de políticas públicas. En: Opera, N. ${ }^{\circ}$, pp. 23-51.

Schell, C.; Riley, C. y Petersen, G. R. (2008). Pathways for Development of a Bio-renewables Industry. En: Bioresource Technology, Vol. 99, N. ${ }^{\circ}$ 12, pp. 5160-5164.

Sudha, P.; Ravindranath, N. H. (1999). Land availability and biomass production potential in India. En: Biomass and Bioenergy, Vol.16, N. ${ }^{\circ}$ 3, pp. 207-221.

Van Thuijl, E. y Deurwaarder, E. P. (2006) European Biofuels Policy in Retrospective. [En linea] Energy Research Centre for Netherlands, Discussion Paper N. ${ }^{\circ}$ 16, <http://www.ecn.nl/ docs/library/report/2006/c06016.pdf>. Consultado: Enero de 2012.

Vian, C. E. F. (2003). Agroindústria Canavieira: Estratégias competitivas e modernização. Campinas: Átomo. 
